Rakenteiden Mekaniikka (Journal of Structural Mechanics)

Vol. 53, No. 2, 2020, pp. 85-99

https://rakenteidenmekaniikka.journal.fi/index

https://doi.org/10.23998/rm.80099

(C) 2020 The Authors

Open access under license CC BY 4.0

\title{
Finite element method modeling of crankshaft axial im- pact measurements
}

\author{
Antti-Jussi Vuotikka ${ }^{1}$, Marko Jokinen, Pasi Halla-aho, Jukka Aho, Antti Mäntylä and \\ Tero Frondelius
}

\begin{abstract}
Summary It has been recently discovered that there is a periodical axial impact phenomenon in a running engine crankshaft. Bending of the shaft causes significant extension of the crankshaft and impact to the engine block through the axial thrust bearing. The aim of this work is to study impact-induced energy fluctuations in a complex-shaped Wärtsilä sixteen vee 32 engine crankshaft by using an explicit finite element method (FEM) during the first $25 \mathrm{~ms}$ after impact. Using the FEM allows us to study real components used in industry, and analyze their dynamics in the transient phase. In conclusion, we found interesting results that can be used as guidelines for a full-scale crankshaft measurement instrumentation plan. The full-scale measurements will be performed later in the Wärtsilä Oy facility at Vaasa, Finland. The main finding is that a substantive amount of energy is trapped in the head region and the first two crank pins of the crankshaft, which can affect crankshaft durability regarding high-cycle fatigue.
\end{abstract}

Key words: impact, crankshaft, Wärtsilä, strain wave, data analysis, explicit FEM

Received: 28 March 2019. Accepted: 27 November 2019. Published online: 30 March 2020.

In memory of Professor Emeritus Tapio Salmi

\section{Introduction}

Frondelius et al. present a comprehensive literature review of medium-speed engine development [1]. The crankshaft is the heart of the engine and this study focuses on impactexcited vibration behavior in this component. The aim of this study is to use a finite element method (FEM) analysis to plan a full-scale measurement instrumentation system. Halla-aho et al. [2] studied counter weight (CW) vibrations utilizing a dedicated measurement device, which is also used to detect axial vibrations of the running engine. A significant amount of measurement data must be collected from the crankshaft due to its complex geometry and the need for high measurement frequency when applied on a full-scale basis. Aho et al. [3] demonstrate how to analyze large amounts of measurement data.

Longitudinal vibration of the crankshaft in a large-bore medium-speed combustion engine has a remarkable effect on the overall dynamics of the powertrain and vibration

\footnotetext{
${ }^{1}$ Corresponding author: antti-jussi.vuotikka@gbw.fi
} 
of the engine [4-6]. Although the firing forces do not directly introduce any longitudinal forces to the crankshaft, they do induce bending of the shaft, causing significant extension and shrinking of long shafts. The shaft is supported by the axial thrust bearing from one end of the engine, which consequently causes impacts of the shaft against the engine block. For this reason, it is important to understand the excitations of the engine and the strain waves traveling in the crankshaft, to ensure reliability of the structure.

It is planned to measure the phenomenon described above by using an impact hammer, to apply short longitudinal impact pulses into the flywheel end of the crankshaft of a Wärtsilä sixteen vee 32 engine (see figures 1(a) and 1(b)). It is [7] shown that longitudinal impacts impart a certain amount of strain energy, and kinetic energy, to the structure. Under ideal conditions, the type of energy fluctuates between these two forms as a function of time. The strain energy is in form of strain waves, which propagate through the medium at the sound speed of the specific material. The kinetic energy can be understood in terms of bare rigid body motion. The study will model a complex geometry, a crankshaft, in the time-domain using a commercial explicit FEM program. The goals are to understand the transient effects of the impact, clarify how impact energy propagates through the complex shaped geometry, and establish a baseline for a full-scale measurement instrumentation plan.

This paper is presented in four sections. Consideration is given to the possible analysis methods and their restrictions in Analysis techniques of the impact phenomenon. The planned measurement setup and employed calculation model are described in Calculation model of measurement setups. We believe that this type of measurement arrangement provides valuable information on how the axial impact phenomenon affects the crankshaft. It is essential to know if it significantly increases the stress values and reduces durability, due to fatigue, in the crankshaft. In Calculation results, we show how and where the calculated impact energy and strain waves fluctuate in the crankshaft as functions of time. Finally, in Conclusion, our conclusions are shown regarding how this study can be used as a basis full-scale the measurement planning.

\section{Analysis techniques of the impact phenomenon}

Since the beginning of the last century, a wide variety of calculation techniques [7-18] have been developed and applied for solving problems related to the propagation of elastic waves, contact during impact, and wave interaction with damage-related discontinuities. The simplest method of modeling the collision problems is analytical Newtonian mechanics. It can be used to study impacts on rigid bodies, but these equations are not suitable, even for low-speed impacts between rigid parts, when significant amount of initial energy is converted into internal energy [16]. One-, two-, and three-dimensional (1D, 2D, and 3D) analytical models have been presented for impact phenomena and wave propagation in elastic materials [17]. These can used if the component under study has a very simple shape, but components in real life rarely have this kind of shape, which hinders the usability of these models. Different types of contact models can be used to model the impact between parts, but St. Venant's and Hertz's models are not very accurate [10]. Coulomb's dry friction formulation of the contact is sufficient to model impact phenomena in contact surfaces [16].

FEM gives the ability to study wave propagation in real components used in industry. FEM techniques can be divided into two main classes [17]: implicit and explicit. The former method is a, so-called, frequency-domain method, which is based on the frequency 
representation of excitation signals and structural responses. The latter is a, so-called, time-domain method. In both methods, the geometry is discretized by using an element mesh. There exist different types of requirements for element mesh, depending on the subject of interest, such as studied frequency band, wavelength, or contact surfaces [15]. The explicit method is usually employed for wave propagation studies, because it is faster than the implicit method.

The Central difference method is employed in this study via implementation into ABAQUS as an Explicit method [19]. The central difference method is conditionally stable [20], which means that the analysis time increment has a critical upper limit. The time increment must satisfy the equation $\Delta t \leq 2 /\left(\omega_{\max }\right)$, which is called Courant's condition. If damping is present in the system, a more complicated condition can be written as, $\Delta t \leq 2 /\left(\omega_{\max }\right) *\left(\sqrt{1+\xi^{2}}-\xi\right) . \omega_{\max }$ is the highest frequency of the system and $\xi$ is the fraction of critical damping on the highest frequency. Damping reduces the size of critical time increment leading to longer simulation times. Usually, it is estimated that the internal damping of steel materials is negligible; thus, the effect of damping is ignored in this study.

The calculation of eigenvalues is not efficient in the explicit method so there is the need of a better estimator for the critical time increment [21]. It is shown that the highest frequency of the element is higher than the frequency of the system, $\omega_{\max }^{\text {sys }} \leq$ $\omega_{\max }^{\text {ele }}$, which leads to a conservative estimate of the time increment, $\Delta t_{\max }^{\text {ele }} \leq \Delta t_{\max }^{\mathrm{sys}}$. ABAQUS [19] calculates the critical time increment for the element by using the equation $\Delta t=\min \left(L_{e} / C_{d}\right)$. The minimum characteristic dimension $L_{e}$ is measured from the all elements and the wave speed is calculated for the current element $c_{d}=\sqrt{(\lambda+2 \mu) / \rho}$, where $\rho, \lambda$ and $\mu$ are material parameters. It can be said that the strain wavefront should not propagate over one element during a time increment.

It can be seen that the cost of explicit simulations is dependent only on mesh size and number of the time steps, but not the frequency range of interest. By using smaller time steps, it is possible to raise the accuracy of results, but simulation time increases. Thus, there are different requirements for time increments to catch studied physical phenomenon properly $[8,9,22]$. It is said that the studied frequency band should limit the size of the time step. If it is necessary to study a frequency band up to $f_{\max }$, the time step $\Delta t$ must be at least $\Delta t=1 /\left(20 f_{\max }\right)$. The smallest studied wavelength $l_{\min }$ also limits the element mesh size $l_{\mathrm{e}}$. It is recommended to use at least 10 elements over the wavelength, which should give reasonable results of the phenomenon, $l_{\mathrm{e}}=l_{\min } / 10$.

\section{Calculation model of measurement setups}

The FG1 impact hammer is chosen as the device to impact the studied structure (see figure 1(a)). The hammer is manufactured by Global Boiler Works Oy. The impact phenomenon can be divided into four different speed classes: low, intermediate, fast, and hyper fast impacts. Hammering is classified as a low-speed impact, because the impact speed remains under $10 \mathrm{~m} / \mathrm{s}$. Impacts can be divided into direct central and noncentral impacts, and they can be of single or multi-collision type [16]. The employed hammer structure gives a single direct central impact to the studied structure.

The FG1 hammer exhibits a very simple working principle. The piston of the hammer is loaded against a spring using pressurized air. When the pressurized air is released, the piston hits the end of the anvil and causes impact to the studied structure. The intensity and length of the impact amplitude are well known, and the impact is highly repeatable. 


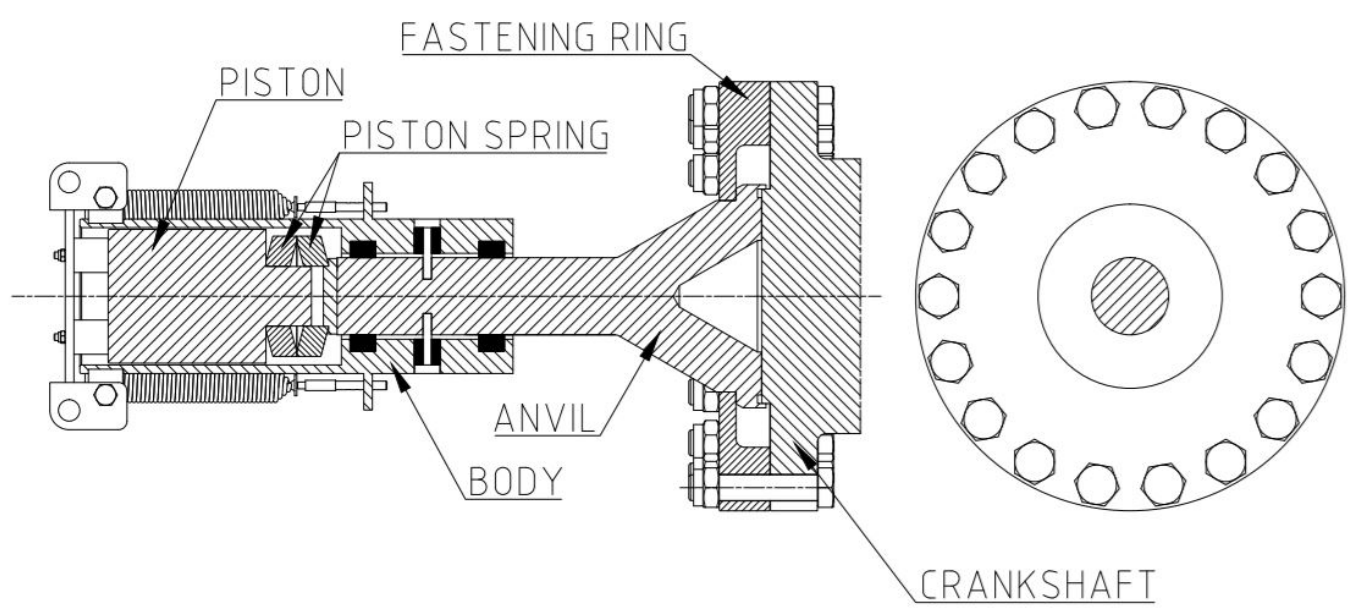

(a) FG1 mounted to the crankshaft.

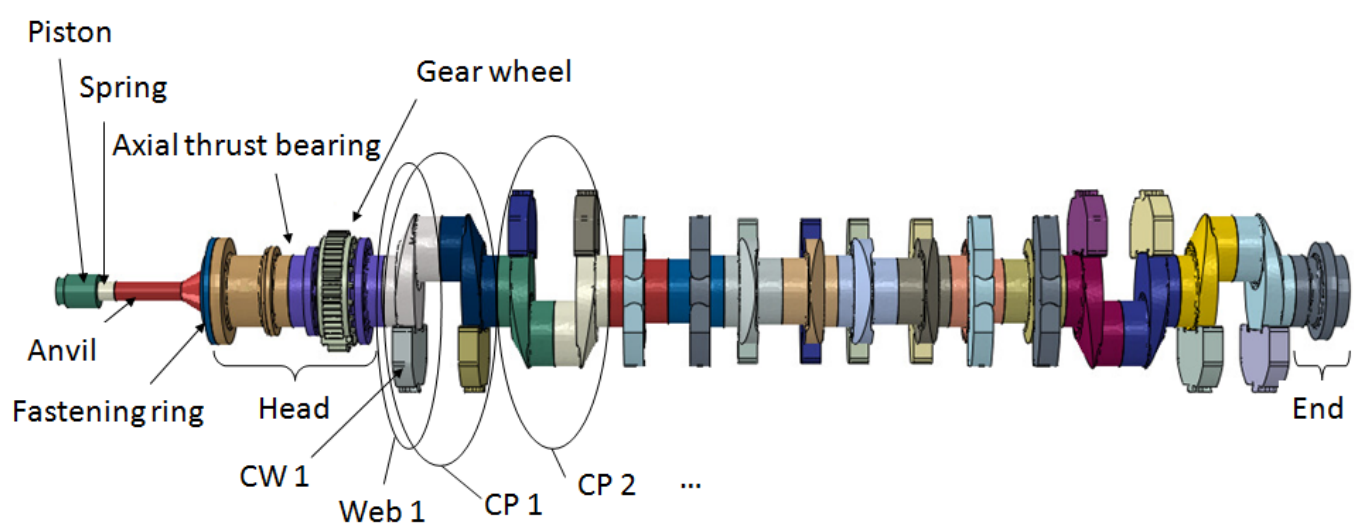

(b) The analyzed crankshaft is divided into different element sets, which are used to study energy distribution in the structure. The two left-most components are the piston and spring, which constitute the hammer. The hammer is followed by an anvil and fastening ring, which attaches the hammer to the crankshaft. The crankshaft is divided into different sections, starting from the head, followed by the crank pins CP1 to CP8, and the end-section. The crank pins can be divided into webs from 1 to 16 . Finally, the counter weights, CW 1 to 16 , are connected to the crankshaft using two bolts.

Figure 1. Schematic diagram of analyzed calculation setup.

Kinetic energy is not conserved during a collision, if the colliding bodies cannot be considered as rigid bodies. Even the piston of the hammer has to be classified as a flexible body, because it contains a spring. The kinetic energy transforms into internal potential energy forms such as strain energy, or it is dissipated into heat based on the hysteresis of the material or frictional contact surfaces. The direct collision of the piston and the measured component ensures that there are no frictional effects on the impact surface.

The old impact hammer type FW2000 has previously been used in wave propagation studies, and is reported in papers [23] and [24]. The first of these papers describes an impact pulse measured from a 6 -m-long bar. The second shows how the measured pulse can be analyzed using ABAQUS Explicit. The measured and modeled impact waves in the steel bar are shown in figure 2. This same impact pulse can be produced with the new type of hammer (FG1) and it is used with the crankshaft impact studies.

The following test setup is planned for the crankshaft measurements. Figure 1(a) 


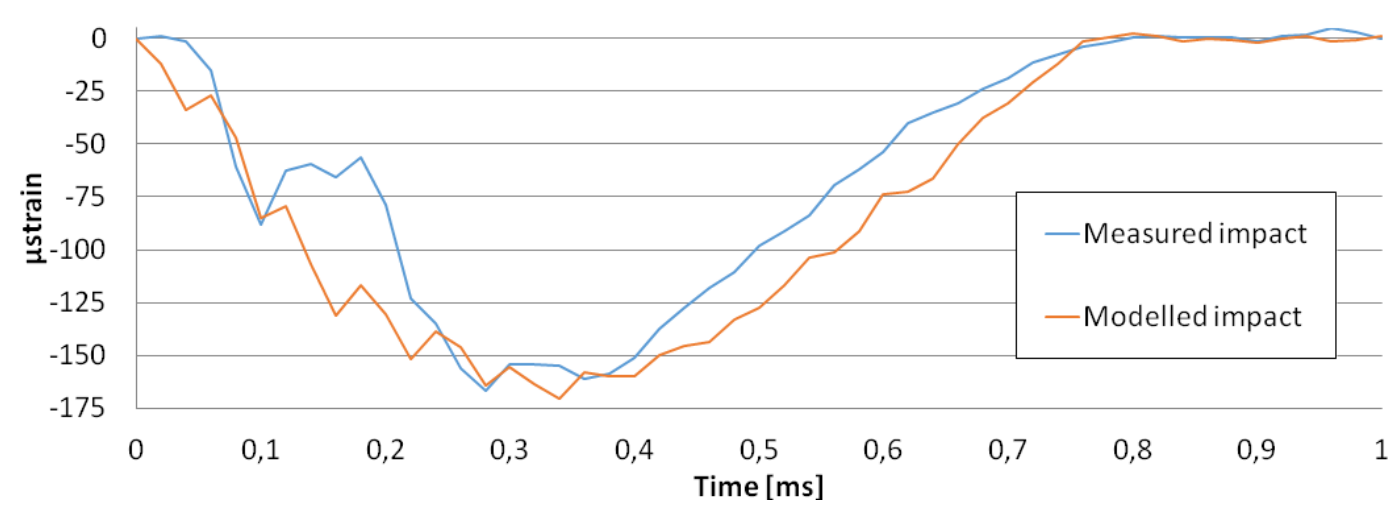

Figure 2. Measured and calculated strain wave from 6-m-long bar. It can be seen that the measured and calculated impacts are nearly equal. The differences are due to the simplified calculation geometry of the piston spring. [23, 24]

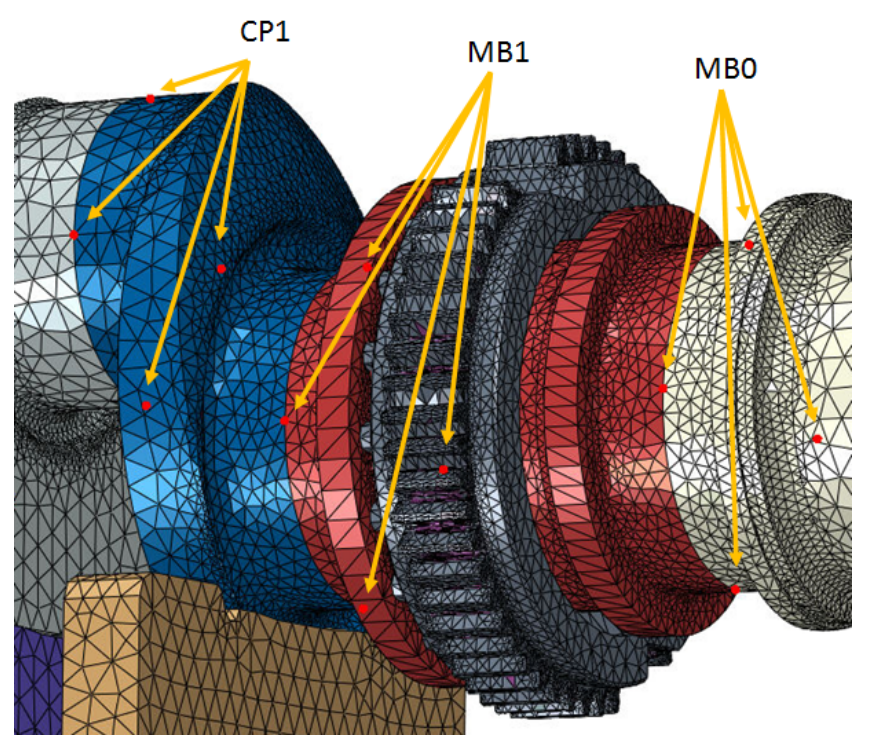

Figure 3. The crank pin and main bearing surfaces have four sample points (red dots) on each surface. The axial strain wave is measured from these points.

shows how the hammer is mounted to the crankshaft using mounting devices: an anvil and a fastening ring with pre-tensioned bolts. The geometry of the hammer and the mounting parts are designed by Global Boiler Works Oy. The geometry of the crankshafts is provided by Wärtsilä $\mathrm{Oy}$.

The hammer body is connected to the anvil the same way it was attached to the steel bar. The hammer body is locked to the anvil by using a locking pin. Sliding surface contact occurs between the hammer body and the anvil side surface. It is assumed that the hammer body does not affect the wave propagation in the anvil and crankshaft, due to sliding surface contact. Thus, the piston and the spring of the hammer are modeled and the body is excluded in the calculation model; see 1(b). The real spring geometry is one pair of Belleville springs. It is replaced by a sleeve in the calculation model, the material properties of which are tuned to obtain the same response as from the Belleville spring.

The piston with spring strikes the free end of the anvil and the fastening ring with bolts keeps the anvil and the crankshaft in contact during the impact. The fastening ring is attached to the crankshaft using pre-tensioned bolts. It is assumed that the pre- 
tensioned bolts do not affect the impact wave propagation thus, the bolts are omitted from the calculation model.

The employed FE calculation program is ABAQUS Explicit, which calculates the problem in the time-domain. The components of the analyzed measurement setup are discretized by using 3D solid elements: The piston, spring, anvil, and fastening ring are meshed using C3D8R elements. The crankshaft is meshed using C3D10M elements due to its geometrical complexity. The total numbers of elements are 14,500 and 1,837,000, respectively; see figure 3 for an understanding of the mesh density. It was determined that all the fillets of the crankshaft would not be meshed smoothly, because that would decrease the element size too much and thus excessively decrease the size of the critical time step.

The crankshaft geometry is partitioned into 19 element sets and all 16 counter weights also form their own individual element sets; see figure 1(b). This is done to follow the distribution of the impact energy throughout the structure.

General contact between the spring and anvil is used and the contact can open after the impact. The standard ABAQUS hard pressure over-closure method is used, which employs a kinematic contact algorithm to minimize the penetration of the surfaces [19]. This predictor/corrector algorithm has no influence on the stable time increment. The kinematic contact algorithm can absorb energy when it strictly enforces contact constraints and conserves momentum. The amount of absorption is dependent on the mesh size. The contact is assumed to be frictionless, because there is only movement in the surface-normal direction.

The anvil and fastening ring are attached to the crankshaft using the TIE-constraint with node-surface formulation. This eliminates all degrees of freedom of the slave surface nodes and couples their motion to the motion of the master surface nodes [19]. The piston and spring are connected using the TIE-constraint, which is also used between the counterweights and crankshaft. All the pre-tensioned bolts are omitted from the calculation model.

The following initial conditions are applied for the hammer and crankshaft. The piston and spring have an initial velocity of $2300 \mathrm{~mm} / \mathrm{s}$; see the impact pulse in figure 2 . The initial velocity was chosen according the experiments and modeling knowledge gained from [23] and [24]. The movement of the crankshaft is not restricted. In reality, the crankshaft is supported by 10 main bearings; thus, gravity does not cause bending of the structure inside the engine block. Due to this, the gravity $9810 \mathrm{~mm} / \mathrm{s}^{2}$ and fixed boundary conditions are omitted from the calculation model.

The piston, anvil, fastening ring, and crankshaft have the material properties of steel: $E=206000 \mathrm{MPa}, \nu=0.3$, and $\rho=7.85 \times 10^{-9} \mathrm{t} / \mathrm{mm}^{3}$. The material properties for the spring have been adjusted to obtain a realistic impact. The following material properties are used for the spring: $E=20000 \mathrm{MPa}, \nu=0.3$, and $\rho=2.7 \times 10^{-9} \mathrm{t} / \mathrm{mm}^{3}$.

Mass scaling is not used to speed up analysis. Therefore, it does not affect the size of the critical time increment. The standard bulk viscosity parameters $\left(b_{1}=0.06\right.$ and $b_{2}=1.2$ ) of ABAQUS are used to damp "ringing" in the highest element frequency and to prevent the element from collapsing in the analysis. Material damping is not introduced, because its effect in rigid steel components is considered to be rather small.

Axial impacts, which are initiated from axial thrust bearing, occur every $17 \mathrm{~ms}$. The analysis time of the hammering impact is slightly longer, $25 \mathrm{~ms}$, to see how much axial impact energy is left in the crankshaft when the next axial impact is initiated. The results are collected at a frequency of fifty thousand measurements per second. This is 
the maximum frequency at which our rugged data acquisition platform is capable. This frequency is used for later validation tests with a real structure.

ABAQUS calculates the stable time increment estimates for each element based on linearization about the initial state. In this particular case, the initial time increment is $1.95202 \times 10^{-8} \mathrm{~s}$, according the smallest element dimension. A fully automatic time incrementation method is used, which increases the employed time increment to $3.334 \times$ $10^{-8} \mathrm{~s}$ during the analysis.

The model, with a total of 4.1M variables, has been decomposed into 120 domains and domain-level parallelization is used. The automatic incrementation of ABAQUS used 751,256 increments to solve the problem described above, which takes a total of approximately $7 \mathrm{~h}$.

\section{Calculation results}

\section{Impact pulse in validation bar}

First, the energy behavior in the impact validation bar is studied. The change in energy during the analysis is shown in figure 4(a). It can be seen that total energy is constant through the analysis. The amount of the artificial energy is negligible. The internal energy of penalty contact and viscous damping creates some damping in the model, which accounts for approximately $1.2 \%$ of the total energy. Thus, it can be said that the model behaved well during the analysis.

Initially, all the energy of the system, which is approximately $80 \mathrm{~J}$, is in the hammer's kinetic energy. The hammer transfers $81 \%$ of its energy to the round bar, because the internal energy of the hammer is negligible after the impact and only $15 \mathrm{~J}$ remains as kinetic energy of the hammer. Half of the energy in the round bar is kinetic and the other half is internal energy, which means elastic energy. At the instant when the impact wave reaches the end of the bar, all the energy is kinetic. The strain wave 2 travels in the bar as the analytical theory predicts, according to the energy results.

\section{Calculated impact in the crankshaft}

The strain wave is induced in the crankshaft using the validated $80 \mathrm{~J}$ impact. The hammer's kinetic energy changes to different types of energy during the analysis, as shown in figure $4(\mathrm{~b})$. The hammer transfers only $10.1 \%$ of its initial kinetic energy to the crankshaft, due to the very large mass difference between the colliding parts. The amount of kinetic energy is $70.7 \mathrm{~J}$ and the internal energy is only $0.75 \mathrm{~J}$ in the hammer after impact. Slightly less than half of the total energy in the crankshaft is in the elastic deformations. Only occasionally, does the elastic internal energy level rise above the kinetic energy level.

The total energy of the analysis has clearly behaved similarly to the validation case, because it remains constant during the analysis. The amount of the dissipation and artificial energy is almost negligible, at $1.8 \%$. At this time, the largest portion of these energies remains as artificial energy. Additionally, the internal energy of penalty contact and viscous damping are present but negligible.

In figures 5 and 6 , only the energy changes in the crankshaft are studied. The fastening ring and anvil are omitted from these results. We then separate the crankshaft into three sets (see figure 5(a)): The energy in the straight parts of the head, the gearwheel and straight part of the end section are combined. The energy in the crank pins is combined without the counter weights. The energy in the counterweights is combined. We can see 


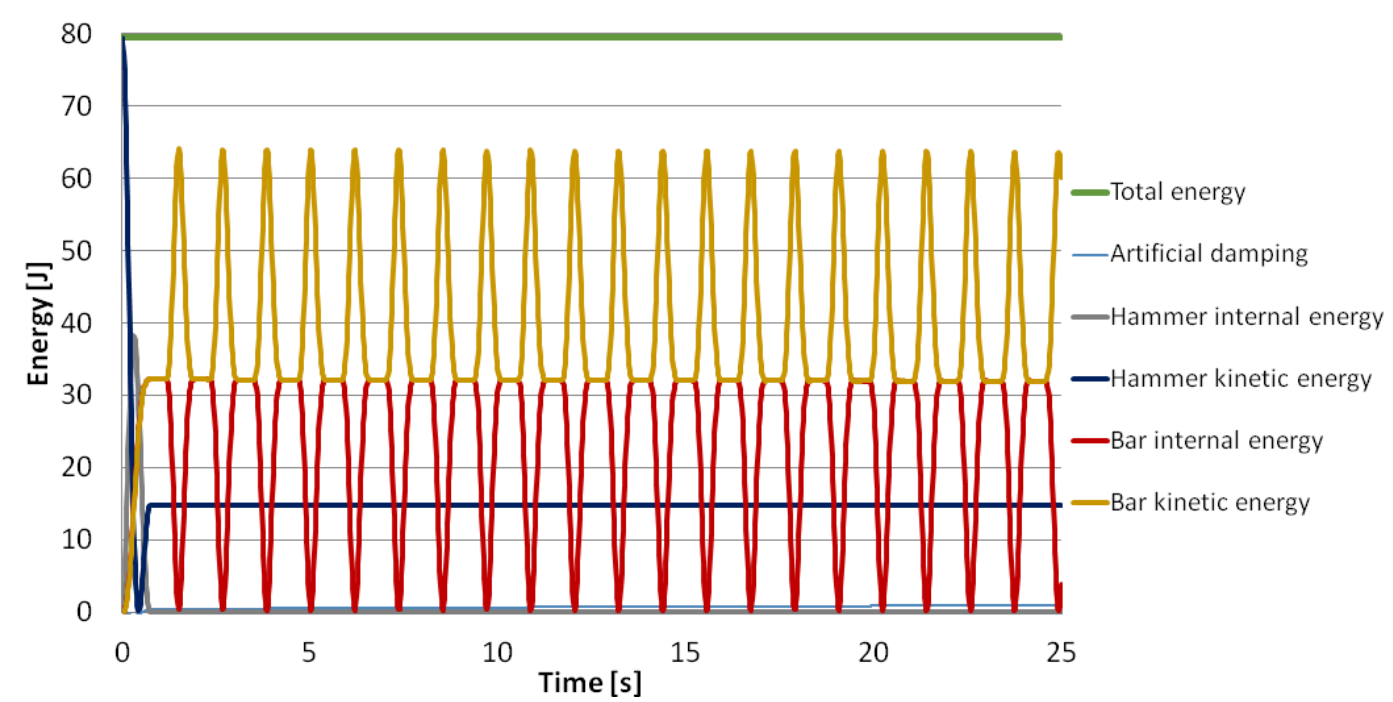

(a) Energy distribution diagram of the validation bar analysis. A small amount of kinetic energy has remained after the impact in the hammer. Most of the energy is transferred to the validation bar, where it fluctuates between kinetic and internal energy.

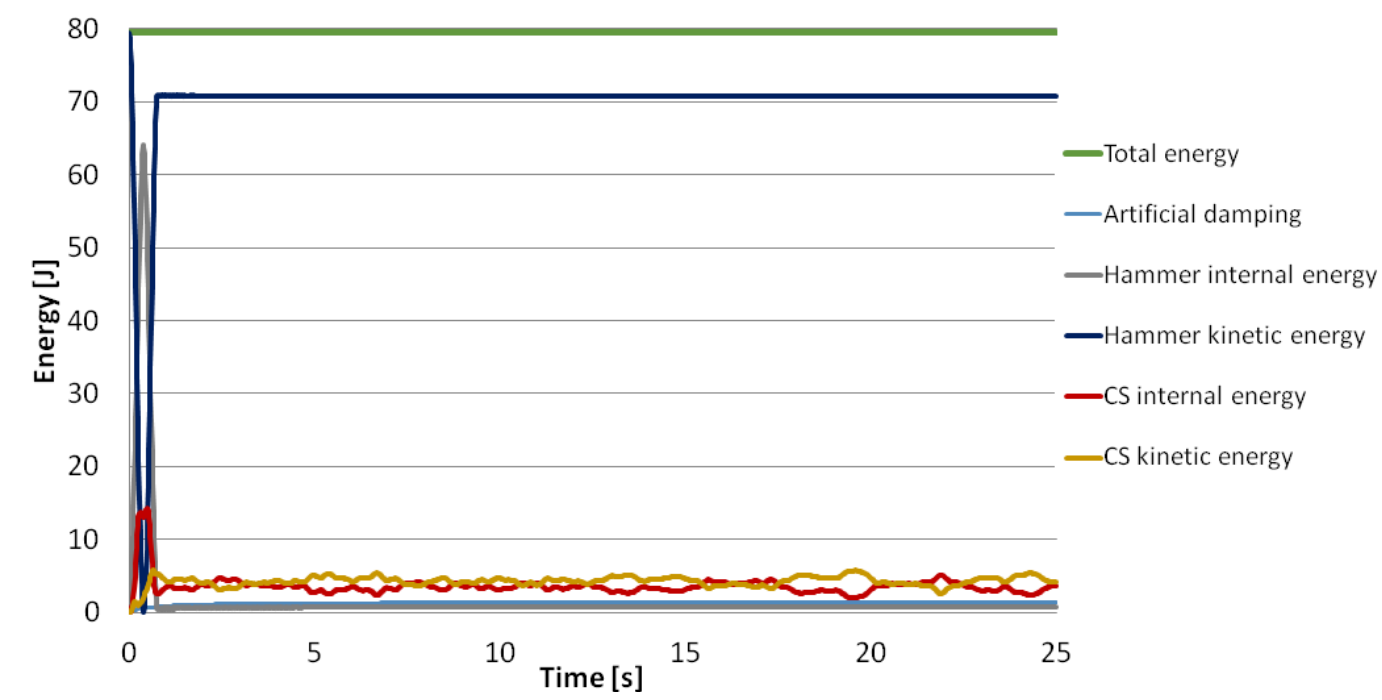

(b) The energy distribution diagram of the crankshaft impact test setup analysis. Only a small amount of the initial kinetic energy of the hammer is transferred to the crankshaft, due to their huge mass difference.

Figure 4. Energy balance of the analysis.

in figure 5(a) that the head has a high energy peak at the beginning of the analysis due to the impact. The duration of the first impact wave is approximately $1 \mathrm{~ms}$ and after that the energy diminishes to only a small fraction of the total energy in the crankshaft. According to this result, there is not a high-intensity standing wave in the head part of the crankshaft, but the impact energy travels further into the crankshaft body. The longitudinal wave propagates to the first web, where the discontinuous geometry with the longitudinal wave initiates flexural waves to the crankshaft. The total energy included in the flexural waves can be divided into the counter weights and webs. The webs contain more energy than the counter weights. They have approximately $55 \%$ and $37 \%$ of the energy, respectively.

In figure 5(a), the total energy is presented for three sections of the crankshaft. Figure 5(b) shows the kinetic and internal energies for these sections. The head section of the 


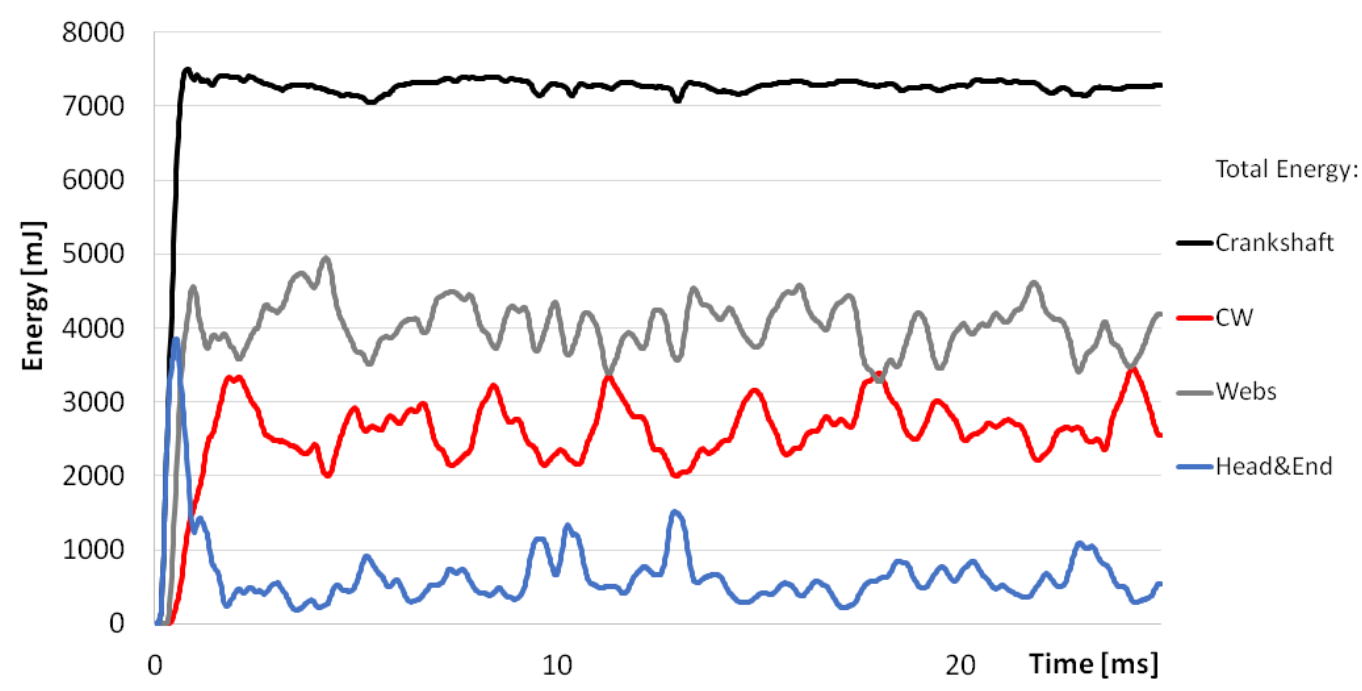

(a) Total energy in crankshaft divided into three different parts: combined counter weights, combined crank pins without counter weights, and combined head and end sections of the crankshaft.

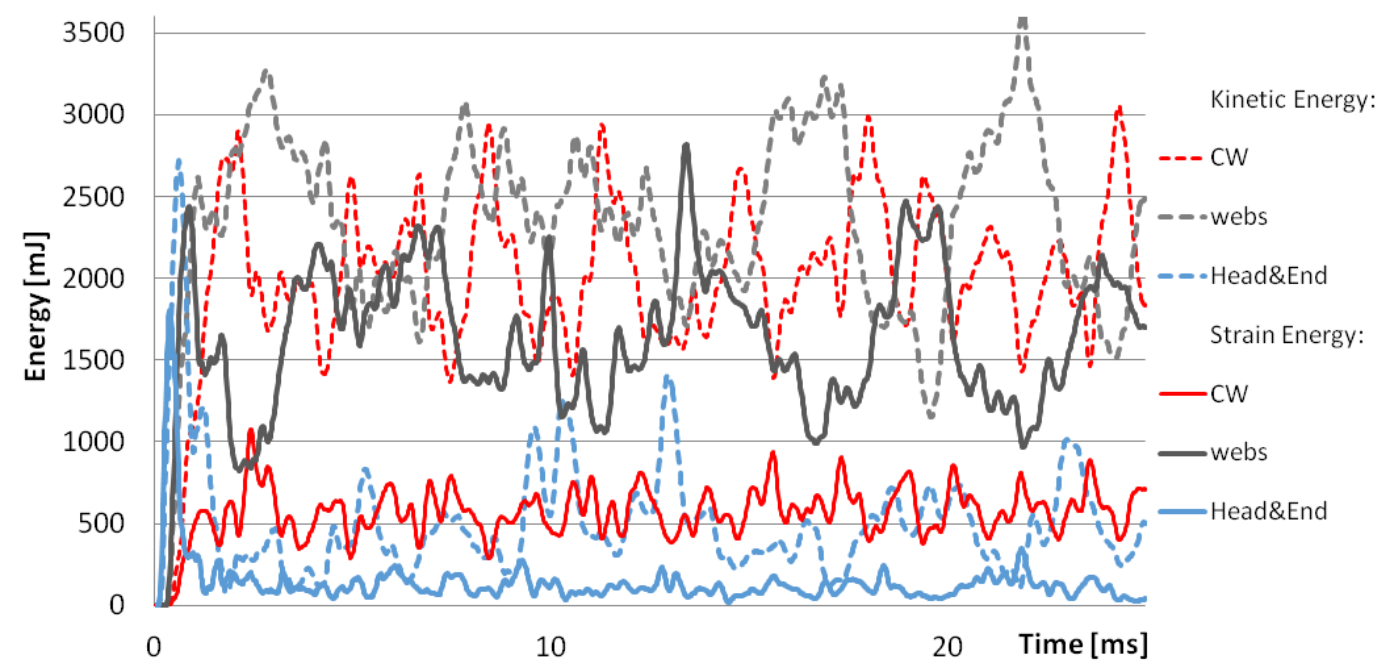

(b) Total energies of the previous figure divided into kinetic and strain forms.

Figure 5. Energy distribution in crankshaft.

crankshaft has an internal strain energy for only a short period just after the impact. The peak value $1.4 \mathrm{~J}$ is gained at $0.4 \mathrm{~ms}$ after the start of the analysis. The strain energy is negligible after $0.7 \mathrm{~ms}$ and the head section mainly exhibits rigid body motion. The same observation can be noted in the counterweight, in which the energy is mainly in the kinetic form, at $2.7 \mathrm{~J}$. This is a rather expected finding, because the counter weights are cantilever parts attached to the crankshaft body. The situation is different in the webs, where the kinetic and strain energies fluctuate and they have almost the same intensity. The internal and kinetic energies are approximately 2.5 and $1.6 \mathrm{~J}$, respectively.

Then total energy is divided between each individual counter weight in figure 6(a). The first and third counter weights have the largest amount of the energy after the impact wave, but it also remains high during the fluctuation. It is surprising that the energy of the second counter weight is clearly lower than that of the first and third CWs, but it is still higher than that of most of the other CWs. The fifth and seventh CWs also 


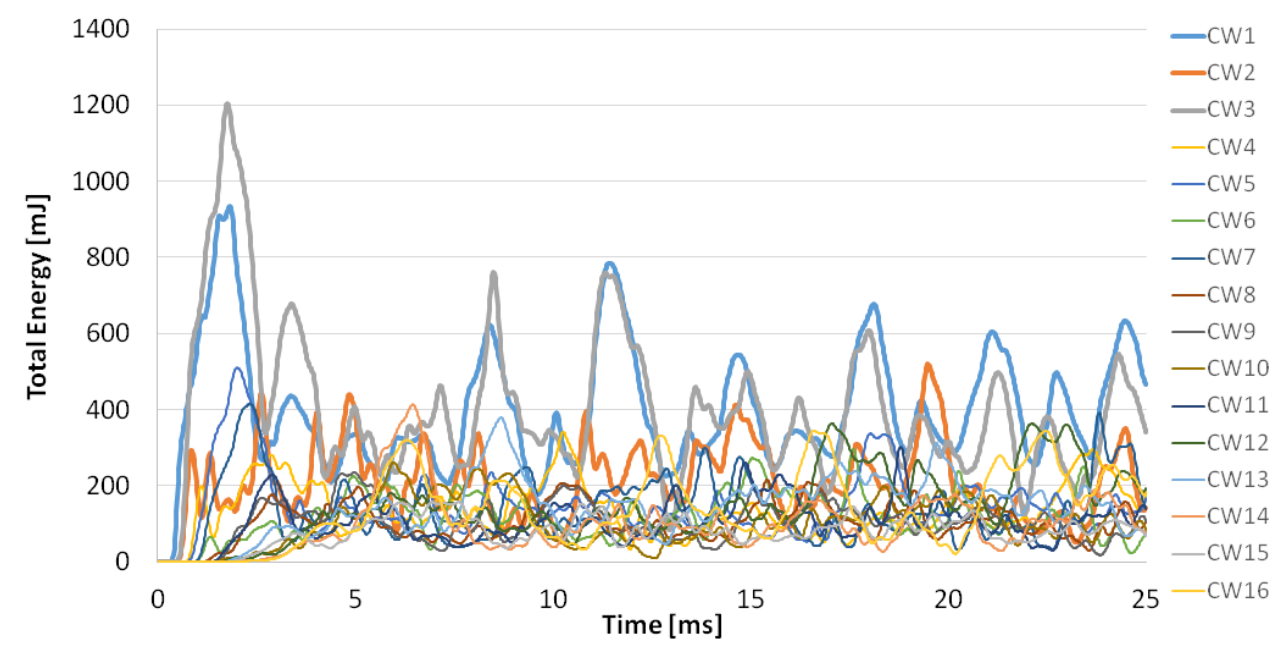

(a) Total energy in counter weights.

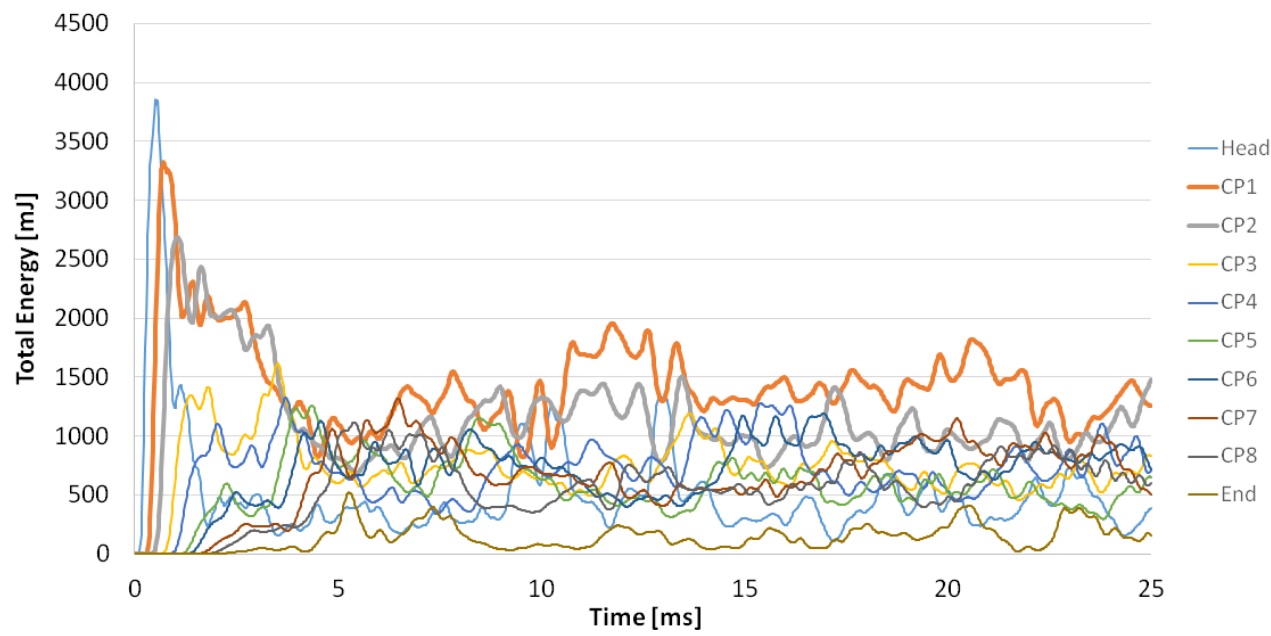

(b) Total energy in the different sections of the crankshaft body. Both counter weights are included in the crank pin results.

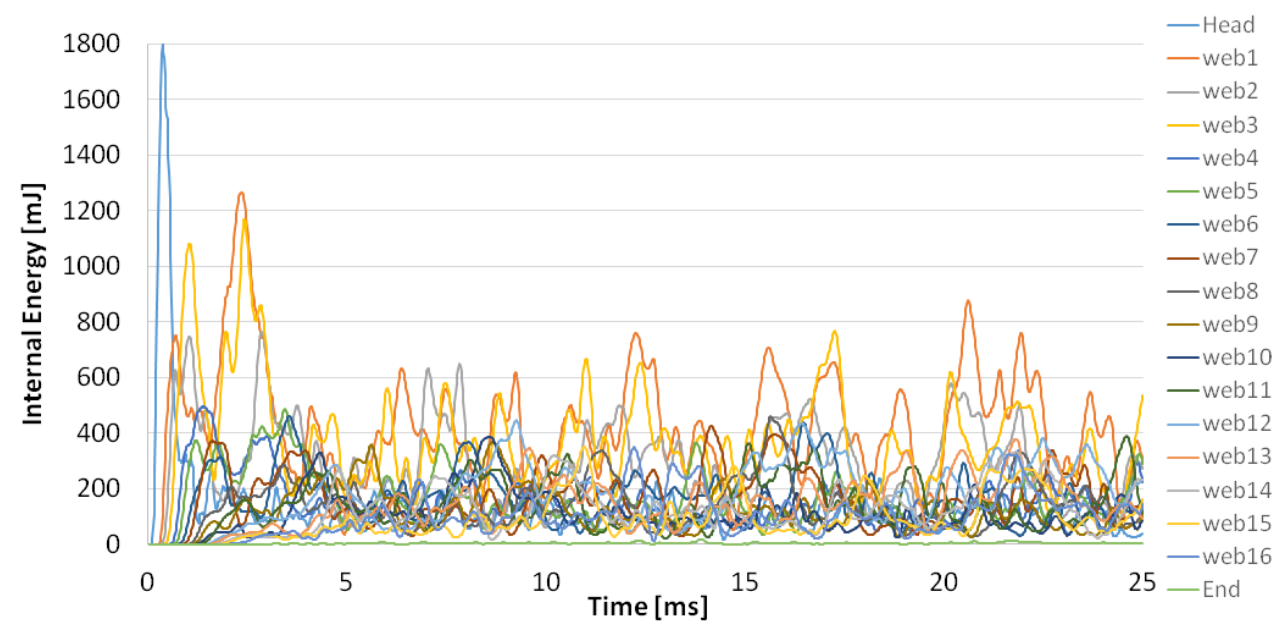

(c) Internal energy of the webs.

Figure 6. Energy distribution in different sections of the crankshaft. 
have rather high energy after the first wave, but the rest of the CWs have energy levels below $0.2 \mathrm{~J}$.

Let us divide the energy in the head section and the crank pins, as shown in figure $6(b)$. Now, we can see how the wave front propagates through the crankshaft. The energy level starts growing sequentially in all sections, every $0.25 \mathrm{~ms}$. The wave reaches the end section $2.5 \mathrm{~ms}$ after the impact. The highest intensity can be predictably found from the head section, because all the impact energy is traveling through that section just after the collision. The high energy level propagates fast through the section and then diminishes. The first two crank pins' energy levels grow rapidly; their energy levels last much longer and the intensity remains higher than in the rest of the crank pins. The median energy level is $0.7 \mathrm{~J}$ in the rest of the crank pins.

Finally, figure 6(c) showing the internal energy in the webs and CW is analyzed. The head part has the highest strain energy intensity of $1.8 \mathrm{~J}$ after the impact. The internal energy wave propagates to the first three webs, which have clearly higher energy states than the rest of webs. The first wave is over $1.0 \mathrm{~J}$ and following waves all have over $0.6 \mathrm{~J}$.

According to these results, it is clear that impact causes highly complex phenomena in the crankshaft. It was not unexpected that such clear periodical behavior is not exhibited as in the straight validation bar. The energy results showed that the following findings can be used when planning measurements: The first two CWs have high kinetic energy levels. Thus, this is a good location to measure accelerations to identify the natural modes that an axial impact can excite. The first main bearing is a good place to measure longitudinal wave propagation in the head section using strain gauges. The first two crank pins have the highest internal energies. The strain gauges should capture strains due to the bending modes of the crankshaft. The results in figure 7 illustrate the level of the strains in the crankshaft.

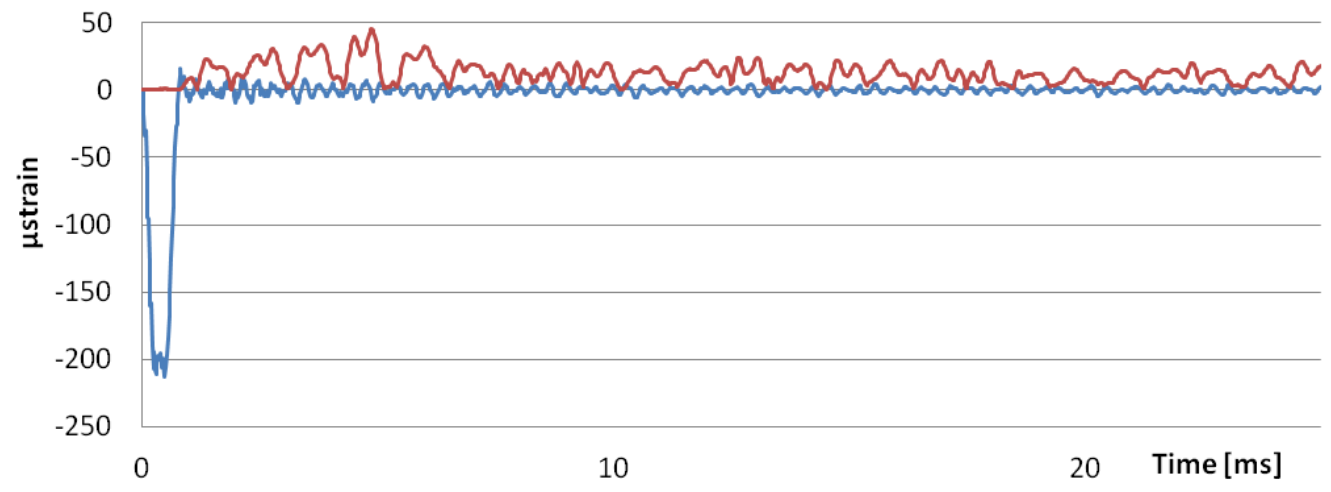

Figure 7. Strain is measured from the anvil. The impact is straight, but reflection waves show the bending behavior.

The impact pulse in the anvil can be seen from figure 7. The axial strain is measured at four points. The mean and amplitude of the strain are calculated according to results from these four points. The points are equidistant from the head of the anvil, and the angle between each pair of adjacent points is 90 degrees. The maximum peak value of the strain in the anvil is $-210 \mu$ strain, which corresponds to a $42 \mathrm{MPa}$ stress state. The impact is straight, because the amplitude is close to zero during the first wave. The mean strain remains low after the first wave. The intensity is mainly below $5 \mu$ strain. The reflected waves from the crankshaft induce bending of the anvil. The maximum strain is 


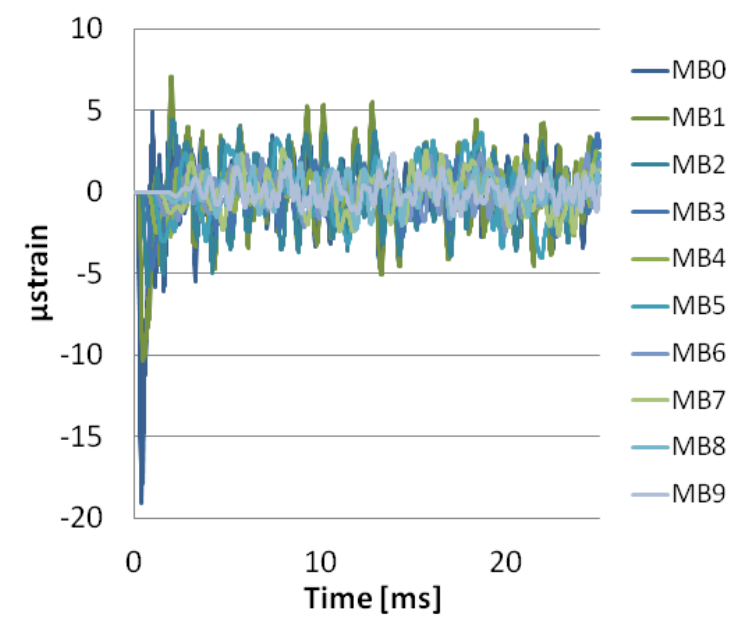

(a) Mean value of axial strain.

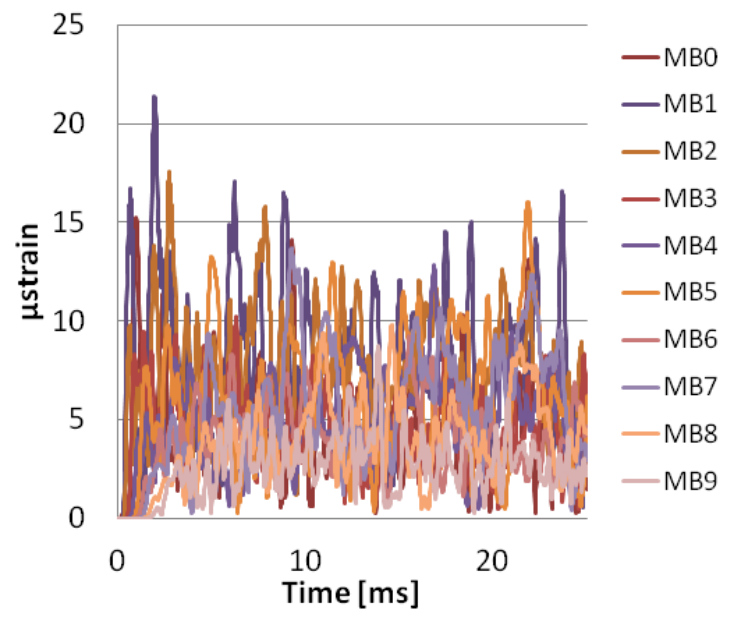

(b) Amplitude of axial strain.

Figure 8. Axial strain in main bearings.

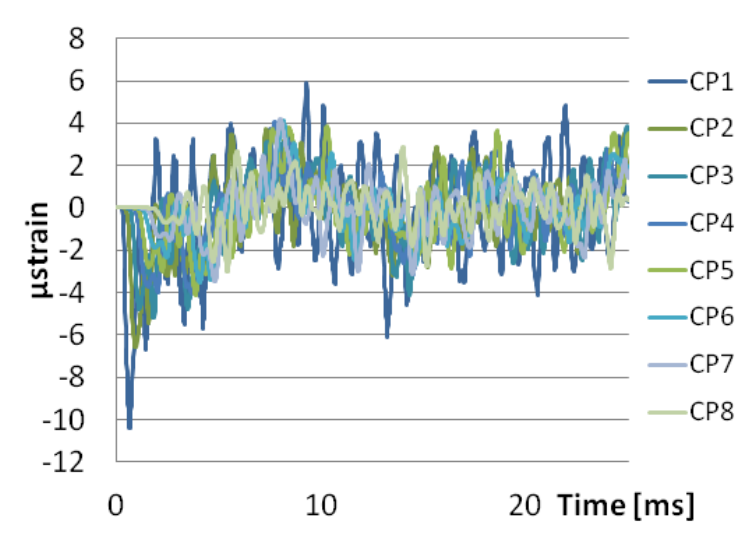

(a) Mean value of axial strain.

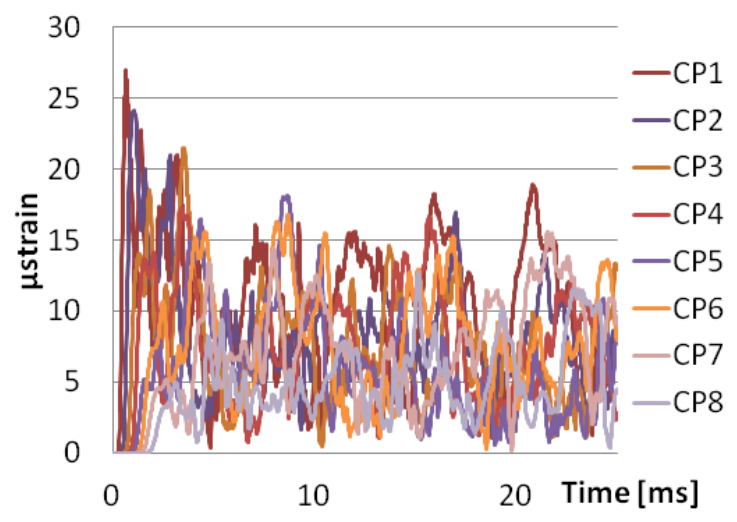

(b) Amplitude of axial strain.

Figure 9. Axial strain in crank pins.

$45 \mu$ strain in the bending waves.

The strain wave propagates to the head of the crankshaft from the anvil. The strain level here is much lower due to the thicker body. MB0 observes a $-19 \mu$ strain compression strain pulse and MB1 only observes $-10 \mu$ strain. The mean strain remains higher in MB1, which has several smaller peak values over $5 \mu$ strain. The third and fourth main bearings have some peaks over $4 \mu$ strain, but the rest remain under that value. The strain amplitude components are highest in MB1, which have a single peak over $20 \mu$ strain strain. There are some peaks over $15 \mu$ strain in MB0, MB1, MB2, and MB5.

From MB1, the impact wave reaches the first crank pin CP1. The compression wave intensity is $-10 \mu$ strain when it passes through the pin. The mean stress peak values remain higher than those of the other crank pins through the analysis. The second and third crank pins have some mean strain waves over $5 \mu$ strain but rest of them remain under $5 \mu$ strain. The bending component is higher in the crank pins than in the main bearings. All the crank pins have over $10 \mu$ strain amplitude strain components, and the highest peaks of the first three pins are over $20 \mu$ strain.

These strain values are obtained from the middle of the bearing surfaces. It is expected that higher strain values are highly localized, and they can be found near the crank pin 
and bearing surface fillets, as well as near the counterweight mounting regions. These absolute values are not so interesting in this analysis, because there should be a much smoother element mesh in the above-mentioned fillets to obtain plausible results.

\section{Conclusion}

The explicit FEM is an excellent method to evaluate the dynamic behavior of components in the time-domain. It is possible to follow how and where the impact energy travels in the component in the transient phase that occurs before the natural modes are finally present in the structure.

As expected, the energy behavior of the validation bar was very simple. This would even be possible to calculate by using the theoretical approach, but it is not possible with real-world complex geometries such as a crankshaft.

The results showed that artificial and damping energies were low in both analyses. According to this, the results are reliable. Of course, we must remember that there are several damping sources in the real engine block, which we could not consider here in the FEM.

The results showed that the highest energy intensity remained in the first two crank pins during the employed analysis time of $25 \mathrm{~ms}$. According to this, the first four main bearings and the first two crank pins are good locations for strain gauges. The energy in the first and third counter weights is mainly in the kinetic form, and thus, at least these should be instrumented using accelerometers. The FEM results showed that strains are measurable and that the strain intensity is high enough that its effect should be studied more carefully with measurements.

The novelty of this study is the modeling of a complex geometry, a crankshaft, in the time-domain. The aim was to obtain a basis for measurement planning, and it can be concluded that the study was a success. As future work, the measurement results should be independently published.

\section{Acknowledgements}

The authors would like to acknowledge the financial support of Business Finland (formerly Tekes) in the form of a research project WIMMA Dnro 1566/31/2015.

\section{References}

[1] Tero Frondelius, Hannu Tienhaara, and Mauri Haataja. History of structural analysis \& dynamics of Wärtsilä medium speed engines. Rakenteiden Mekaniikka, 51(2):1-31, 2018. open access. URL: https://doi.org/10.23998/rm.69735.

[2] Pasi Halla-aho, Antti Mäntylä, Tero Frondelius, Tommi Helander, and Juha Hautala. Counterweight measurements device development. Rakenteiden Mekaniikka, 50(3):318-322, 2017. open access. URL: https://doi.org/10.23998/rm.65050.

[3] Jukka Aho and Tero Frondelius. Analyzing 3 TB field measurement data set. Rakenteiden Mekaniikka, 50(3):224-228, 2017. open access. URL: https://doi.org/ $10.23998 / \mathrm{rm} .64942$. 
[4] Ilkka Väisänen, Antti Mäntylä, Antti Korpela, Teemu Kuivaniemi, and Tero Frondelius. Medium speed engine crankshaft analysis. Rakenteiden Mekaniikka, 50(3):341-344, 2017. open access. URL: https://doi.org/10.23998/rm.64916.

[5] Tero Frondelius, Pasi Halla-aho, and Antti Mäntylä. Crankshaft development with virtual engine modelling. In CIMAC Congress Helsinki, 2016.

[6] Rune Nordrik and Haavard Solbakken. Vibration characteristics of a v20 medium speed gas engine. simulation and measurement. In CIMAC, CIMAC World Congress on Combustion Engine, 26th, Bergen, NO, 2010-06-14 - 2010-06-17, 2010.

[7] Herbert Kolsky. Stress waves in solids, volume 1098. Courier Corporation, 1963.

[8] D Cerniglia, A Pantano, and N Montinaro. 3d simulations and experiments of guided wave propagation in adhesively bonded multi-layered structures. NDT $\&$ E International, 43(6):527-535, 2010. URL: https://doi.org/10.1016/j.ndteint. 2010. 05.009 .

[9] Emmanuel Le Clezio, Mihai Valentin Predoi, Michel Castaings, Bernard Hosten, and Martine Rousseau. Numerical predictions and experiments on the free-plate edge mode. Ultrasonics, 41(1):25-40, 2003. URL: https://doi.org/10.1016/ S0041-624X (02) 00391-8.

[10] Bin Hu, Werner Schiehlen, and Peter Eberhard. Comparison of analytical and experimental results for longitudinal impacts on elastic rods. Modal Analysis, 9(1-2):157174, 2003. URL: https://doi.org/10.1177/107754603030745.

[11] Bin Hu and Peter Eberhard. Symbolic computation of longitudinal impact waves. Computer methods in applied mechanics and engineering, 190(37):4805-4815, 2001. URL: https://doi.org/10.1016/S0045-7825(00)00348-0.

[12] Huang Wei and Zou Yida. Finite element analysis on collision between two moving elastic bodies at low velocities. Computers $\& 5$ structures, 57(3):379-382, 1995. URL: https://doi.org/10.1016/0045-7949(95)00046-J.

[13] VD Kubenko. Local wave theory of the collision of elastic bodies. noncentral impact - two-dimensional problem in the ideal-fluid approximation. International Applied Mechanics, 35(11):1155-1166, 1999. URL: https://doi.org/10.1007/BF02682361.

[14] Svetlana Polukoshko, Janis Viba, Olga Kononova, and Svetlana Sokolova. Rigid body impact models partially considering deformation. Estonian Journal of Engineering, 13(2), 2007. URL: http://www.kirj.ee/public/Engineering/2007/issue_ 2/eng_2007_2_7.pdf.

[15] Anthony Sanders, Ira Tibbitts, Deepika Kakarla, Stephanie Siskey, Jorge Ochoa, Kevin Ong, and Rebecca Brannon. Contact mechanics of impacting slender rods: Measurement and analysis. Dynamic Behavior of Materials, Volume 1, pages 229236, 2011. URL: https://doi.org/10.1007/978-1-4614-0216-9_34.

[16] Dan Stoianovici and Yildirim Hurmuzlu. A critical study of the applicability of rigid-body collision theory. TRANSACTIONS-AMERICAN SOCIETY OF MECHANICAL ENGINEERS JOURNAL OF APPLIED MECHANICS, 63:307-316, 1996. URL: https://doi.org/10.1115/1.2788865. 
[17] A Żak and M Krawczuk. Assessment of flexural beam behaviour theories used for dynamics and wave propagation problems. Journal of Sound and Vibration, 331(26):5715-5731, 2012. URL: https://doi.org/10.1016/j.jsv.2012.07.034.

[18] Meng Zhang, Pingsheng Yang, and Yuxu Tan. Micromechanisms of fatigue crack nucleation and short crack growth in a low carbon steel under low cycle impact fatigue loading. International Journal of fatigue, 21(8):823-830, 1999. URL: https: //doi.org/10.1016/S0142-1123(99)00031-6.

[19] ABAQUS v.6.14-2 Commercial FE Software and Documentation. Dassault Systemes, Simulia Corporation, Providence, RI, USA., 2014.

[20] Shen $\mathrm{R} \mathrm{Wu}$ and Lei $\mathrm{Gu}$. Introduction to the explicit finite element method for nonlinear transient dynamics. Wiley Online Library, 2012. URL: DOI:10.1002/ 9781118382011.

[21] DP Flanagan and T Belytschko. Simultaneous relaxation in structural dynamics. Journal of the Engineering Mechanics Division, 107(6):1039-1055, 1981.

[22] Friedrich Moser, Laurence J Jacobs, and Jianmin Qu. Modeling elastic wave propagation in waveguides with the finite element method. Ndt $\&$ E International, 32(4):225234, 1999. URL: https://doi.org/10.1016/S0963-8695(98)00045-0.

[23] M. Jokinen, A.-J. Vuotikka, and S.-G. Sjölind. Suorassa pyörötangossa etenevän jännitysaallon kokeellinen mittaus. In: Mäkinen, $R$. et al.(toim.). Proceedings ot the 10th Finnish Mechanics Days, X Suomen Mekaniikkapäivät, Jyväskylä, 3.-4.12. 2009, 2009.

[24] A.-J. Vuotikka, M. Jokinen, and S.-G. Sjölind. Suorassa pyörötangossa etenevän jännitysaallon analysointi FEM:llä. In: Mäkinen, R. et al.(toim.). Proceedings ot the 10th Finnish Mechanics Days, X Suomen Mekaniikkapäivät, Jyväskylä, 3.-4.12. 2009, 2009.

Pasi Halla-aho, Antti Mäntylä, Tero Frondelius

Wärtsilä

Järvikatu 2-4

65100 Vaasa

firstname.lastname@wartsila.com

Marko Jokinen, Antti-Jussi Vuotikka

Global Boiler Works Oy

Lumijoentie 8

firstname.lastname@gbw.fi

Jukka Aho

ahojukka5@gmail.com

Tero Frondelius

University of Oulu

Erkki Koiso-Kanttilan katu 1

90014 OULUN YLIOPISTO

firstname.lastname@oulu.fi 